\title{
Painless Gross Hematuria: A New Presentation of Primitive Neuroectodermal Tumor of the Prostate
}

\author{
Babak Javanmard ${ }^{1}$, Morteza Fallah Karkan (iD ${ }^{1,}{ }^{*}$, Mohammad Reza Yousefi ${ }^{1}$ and Mahsa Ahadi $^{2}$ \\ ${ }^{1}$ Department of Urology, Shohada-e-Tajrish Hospital, Shahid Beheshti University of Medical Sciences, Tehran, Iran \\ ${ }^{2}$ Department of Pathology, Shohada-e-Tajrish Hospital, Shahid Beheshti University of Medical Sciences, Tehran, Iran \\ "Corresponding author: Resident of Urology, Laser Application in Medical Sciences Research Center, Shahid Beheshti University of Medical Sciences, Tehran, Iran. Tel: \\ +98-2122736386, Email: mortezafallah.md@gmail.com
}

Received 2018 November 12; Revised 2018 December 01; Accepted 2018 December 30.

\begin{abstract}
Introduction: Primitive neuroectodermal tumor (PNET) is a very rare type of prostate sarcoma that mostly occurs in young adults, and it is associated with a poor prognosis.

Case Presentation: A 37-year-old male was admitted with intermittent painless gross hematuria from 1 month prior to admission. Cystosocopy, abdominopelvic computed tomography scan (ACTS), and magnetic resonance imaging (MRI) revealed huge prostate. All tumor markers were negative and pathology findings of trans-rectal ultrasonographic biopsy (TRUS Bx) and trans-urethral resection of prostate (TURP) were consistent with severely inflamed prostatic urethra with no evidence of malignancy. The patient underwent radical prostatectomy. Histopathology of the specimens showed malignant neoplasm of small round and oval cells suggestive of PNET. Immunohistochemistry (IHC) study results on CD99 were positive.

Conclusions: We report a rare uncommon case of prostate PNET presented by intermittent painless gross hematuria. As the prognosis is very poor, medical staff should pay enough attention to the differential diagnosis, choosing the best treatment and subjects close follow-up.
\end{abstract}

Keywords: Primitive Neuroectodermal Tumor, Extra-Skeletal Ewing's Sarcoma, Prostate Sarcoma, CD99

\section{Introduction}

Gross hematuria has a wide differential diagnosis (14). Primary prostate sarcoma, which derives from the mesenchymal cells of the prostate stroma and is responsible for less than $0.1 \%$ of primary prostate cancers, was first reported in 2003. Primitive neuroectodermal tumor (PNET) or extra-skeletal Ewing's sarcoma is a very rare type of prostate sarcoma that mostly occurs in young adults, and is associated with a poor prognosis (5-7). PNET is classified to central and peripheral types according to their locations (3). Cluster designation (CD) 99 positivity distinguishes PNET from other entities such as rhabdomyosarcoma, lymphoma, and neuroendocrine cancer (8).

\section{Case Presentation}

A 37-year-old male was admitted to emergency department with intermittent painless gross hematuria from 1 month prior to admission. The medical and familial histories were unremarkable. He had no documentation of previous malignancies. Digital rectal examination demonstrated a very large prostate with poor mobility. There were no palpable lymph nodes. Ultrasonographic evaluations revealed normal kidney structure, bilateral hydronephrosis, distended Bladder filled with clot, and a huge prostate. According to orthostatic blood pressure changes and hemoglobin of $7 \mathrm{mg} / \mathrm{dL}$, conservative therapy initiated and patient was transferred to operation room (OR) urgently. Cystoscopy and bladder washing was done and bleeding origin was detected from prostate lodge.

The day after that, the patient was reevaluated in OR because of the persistent bleeding. Trans-urethral resection of prostate (TURP) (3 bites for tissue sampling), fulguration, bilateral ureteroscopy, and rectoscopy were performed and, finally, TRUS Bx of prostate was done. The patient's serum prostate-specific antigen (PSA) level was 1.07 $\mathrm{ng} / \mathrm{mL}$, and all of the other serum tumor markers (such as carbohydrate antigen 19 - 9, carcino-embryonic antigen, alpha-fetoprotein, and lactate dehydrogenase) were normal.

Histological findings were consistent with severely inflamed prostatic urethra, papillary heperplasia, and reactive atypia with no evidence of malignancy. The PSA and immunohistochemistry (IHC) results of alpha-methylacyl- 
CoA racemase (AMACR) were negative and leukocyte common antigen (LCA) markers were positive in suspicious cells.

After this report and persistency of gross hematuria, computed tomography (CT) angiography for rule out of arterio-venous malformations, abdominopelvic computed tomography scan (ACTS), and magnetic resonance imaging (MRI) with intravenous contrast were done. All of them showed an $86 \times 57 \times 78 \mathrm{~mm}$ mass replacing the prostate gland, which appeared multi-lobulated nodular mass with central septation (Figure 1).

The patient subsequently underwent radical prostatectomy. Histopathology of the specimens showed prostatic tissue involved by a malignant neoplasm of small round and oval cells with scant cytoplasm suggestive of PNET(Figure 2). IHC study results on CD99 were strongly positive. Also vimentin, B-cell lymphoma 2 (BCL2), and Ki67 were positive, and CD 3, 10, 20 and 34, cytokeratins (CK) 7 and 20, CD117, high molecular weight cytokeratin (HMWCK), PanCK, progesterone receptor (PR), desmin, smooth muscle actin (SMA), S100, terminal deoxynucleotide transferase (TDT), epithelial membrane antigen (EMA), neuron specific enolase (NSE), and synaptophysin were negative in the sample.

According to definitive diagnosis and urethral involvement by tumor adjuvant chemotherapy with ifosfamide ( 2 $\mathrm{mg} / \mathrm{m}^{2} /$ week) started for the patient. CT was rechecked, displaying no evidence of distant metastasis till 9 months after surgery. In the 10th month, the patient referred with the symptoms of abdominal pain and severs constipation, in the abdominopelvic CT scan of a pelvic. A $32 \times 75 \times 112$ $\mathrm{mm}$ mass in the lodge of prostate was seen. The patient was again subjected to 10 sessions of chemotherapy with the previous regiment, but adequate response was not obtained. The patient did not accept redo surgery and died 16 months after the initial operation.

\section{Discussion}

Diagnosis of PNET in the prostate is most challenging. In all prostate PNET case reports, a large-sized primary tumor is seen replacing the prostate lodge. The subjects often described the same sign and symptom as the patients with benign prostatic enlargement (9), whereas our patient presented with gross hematuria. Thus, prostate PNET should be suspected when young males refer with the complaints similar to those of benign prostatic hyperplasia, change of bowel habit or gross hematuria. The ACTS and MRI findings consist of the evidence of a heterogeneously enhanced mass in all cases. And subsequent biopsy with IHC staining is required to confirm the diagnosis $(10,11)$. However, the acceptable management for this
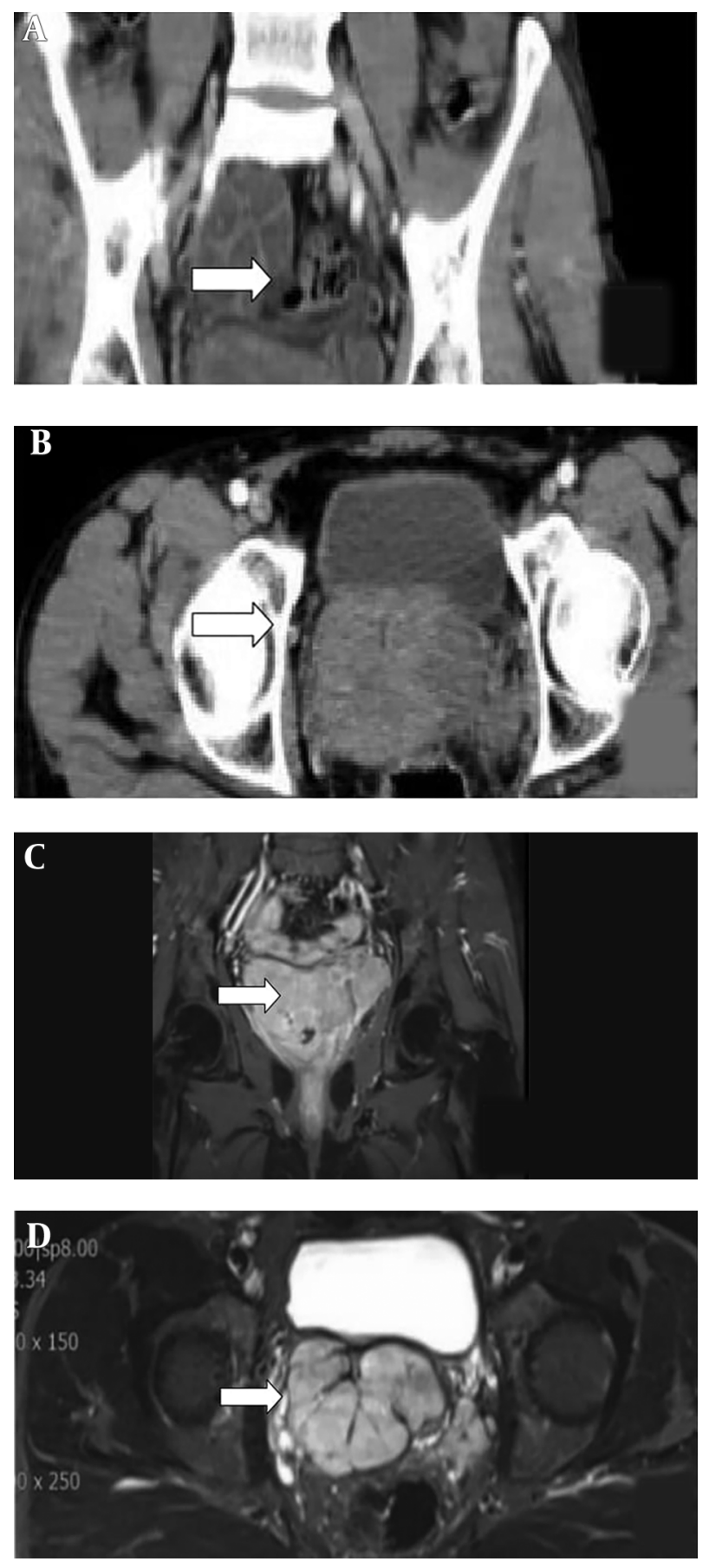

Figure 1. A and B, abdominopelvic CT scan showing an $86 \times 57 \times 78-\mathrm{mm}$ mass replacing the prostate gland (white arrow). $\mathrm{C}$ and $\mathrm{D}$, a magnetic resonance imaging T2-weighted image: There was a multinodular and multilobulated tumor, which replaced the prostate (white arrow).

entity is still debatable. The currently recommended treatment is based on chemotherapy followed by radical prosta- 

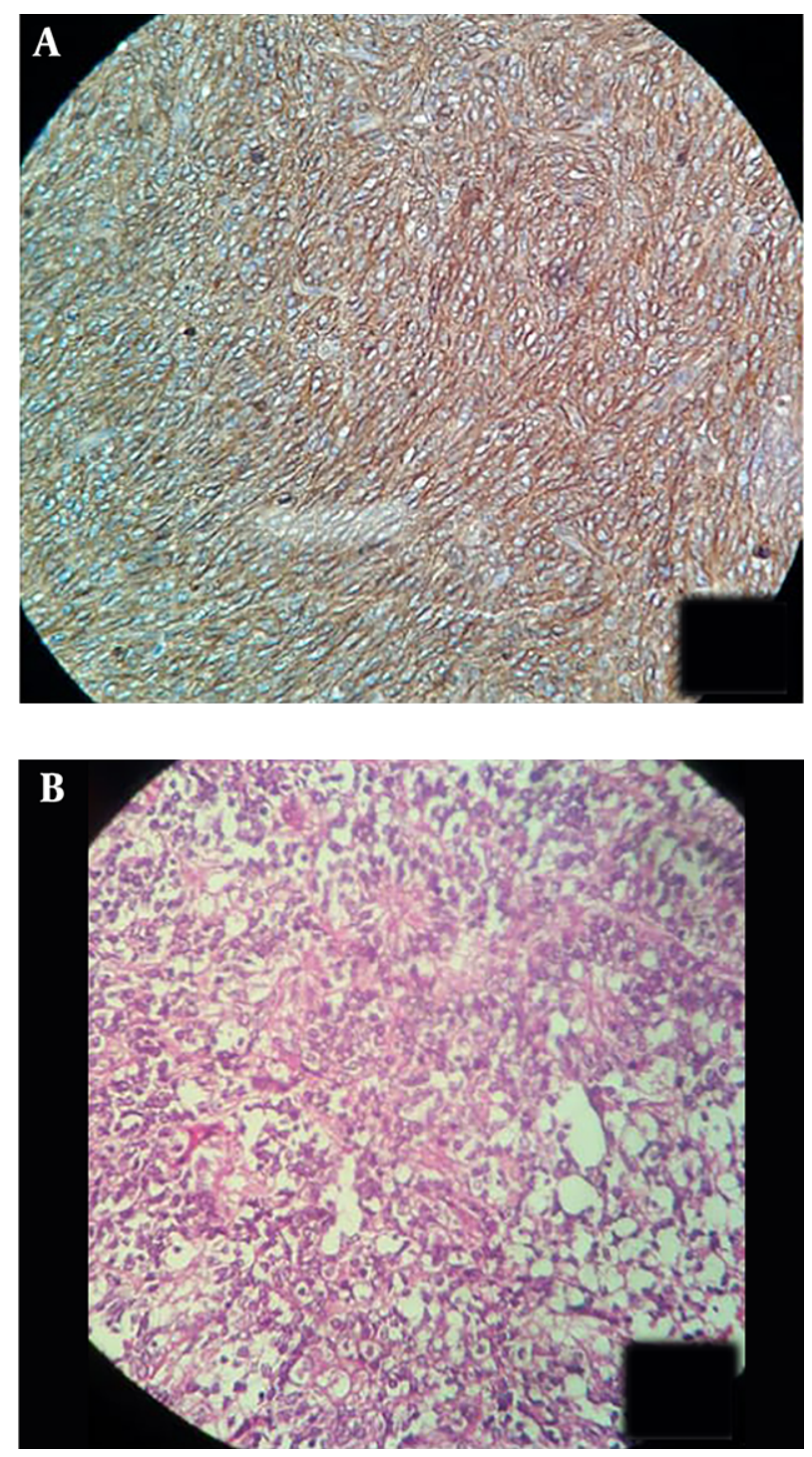

Figure 2. A, immunoperoxidase staining: Membrane-associated CD99 reactivity (CD99 antibody, original magnification $\times 400$ ). B, hematoxylin-eosin staining: Small and similar round cells with round to oval nuclei and scant cytoplasm (original magnification $\times 400$ ).

tectomy/cystoprostatectomy, and radiotherapy. However, data on the long-term follow-up have been limited; most patients were disease-free no more than 24 months after treatment (6).

Including present case, there have been only less than 10 case reports to date of prostate PNET. We report a rare, uncommon case of prostate PNET presented by intermittent painless gross hematuria. As the prognosis is very poor, medical staff should pay enough attention to the differential diagnosis, choosing the best treatment, and subjects close follow up.

\section{Acknowledgments}

None declared.

\section{Footnotes}

Authors' Contribution: None declared.

Conflict of Interests: None declared.

Financial Disclosure: None declared.

Funding/Support: None declared.

Patient Consent: Written informed consent was obtained from the patient.

\section{References}

1. Allameh F, Fallah Karkan M, Nilipour Y, Rakhshan A. Primary signet-ring cell carcinoma of the urinary bladder successfully managed with radical cystectomy in a young patient. Case Rep Urol. 2017;2017:9121078. doi: 10.1155/2017/9121078. [PubMed: 28656119]. [PubMed Central: PMC5474534].

2. Javanmard B, Yousefi MR, Fadavi B, Fallah Karkan M. Retained surgical gauze presenting with gross hematuria: A case report. Urol J. 2017;14(5):5027-9. [PubMed: 28853110].

3. Javanmard B, Haghighat Khah H, Fallah-Karkan M, Khan S. Recurrent hematuria in renal angio-venous malformation, delay diagnosis and endovascular treatment, a case report. Urol Case Rep. 2018;16:46-7. doi: 10.1016/j.eucr.2017.09.008. [PubMed: 29134176]. [PubMed Central: PMC5671406].

4. Allameh F, Najafi S, Fallah Karkan M, Haghighatkhah H, Nekuie S. Chromophobe renal cell carcinoma presented with wunderlich syndrome. Int J Cancer Manag. 2017;10(11). e8057. doi:10.5812/ijcm.8057.

5. Peyromaure M, Vieillefond A, Boucher E, De Pinieux G, Beuzeboc P, Debre B, et al. Primitive neuroectodermal tumor of the prostate. J Urol. 2003;170(1):182-3. doi: 10.1097/01.ju.0000065880.06201.5c. [PubMed: 12796679].

6. Wu T, Jin T, Luo D, Chen L, Li X. Ewing's sarcoma/primitive neuroectodermal tumour of the prostate: A case report and literature review. Can Urol Assoc J. 2013;7(5-6):E458-9. doi: 10.5489/cuaj.1393. [PubMed: 23826070]. [PubMed Central: PMC3699105].

7. Shibuya T, Mori K, Sumino Y, Sato F, Mimata H. Rapidly progressive primitive neuroectodermal tumor of the prostate: A case report and review of the literature. Oncol Lett. 2015;9(2):634-6. doi: 10.3892/ol.2014.2731. [PubMed: 25624891]. [PubMed Central: PMC4301542].

8. Parham DM, Roloson GJ, Feely M, Green DM, Bridge JA, Beckwith JB. Primary malignant neuroepithelial tumors of the kidney: A clinicopathologic analysis of 146 adult and pediatric cases from the National Wilms' Tumor Study Group Pathology Center. Am J Surg Pathol. 2001;25(2):133-46. doi: 10.1097/00000478-200102000-00001. [PubMed: 11176062].

9. Funahashi Y, Yoshino Y, Hattori R. Ewing's sarcoma/primitive neuroectodermal tumor of the prostate. Int J Urol. 2009;16(9):769. doi: 10.1111/j.1442-2042.2009.02339.x. [PubMed:19769660].

10. Atas E, Kesik V. Antracyclin toxicity in a child with primitive neuroectodermal tumor of the chest wall with and brain metastasis. J Cancer Res Ther. 2015;11(3):668. doi: 10.4103/0973-1482.144644. [PubMed: 26458714].

11. Thete N, Rastogi D, Arya S, Singh A, Rao P, Chandge A, et al. Primitive neuroectodermal tumour of the prostate gland: Ultrasound and MRI findings. Br J Radiol. 2007;80(956):e180-3. doi: 10.1259/bjr/57293350. [PubMed: 17762052]. 